\title{
Cancer and Obesity: \\ Am I Part of the Problem?
}

PAMELA HALLQUIST VIALE, RN, MS, CNS, ANP

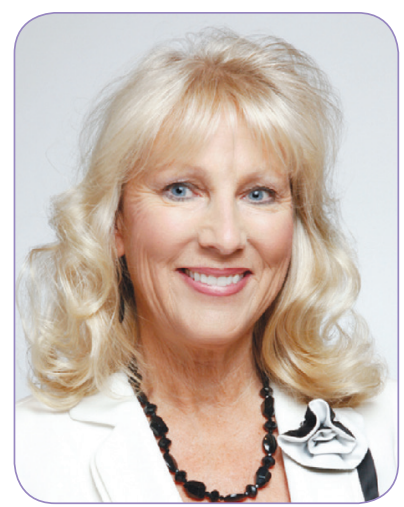

J Adv Pract Oncol 2018:9(6):579-580 https://doi.org/10.6004/jadpro.2018.9.6.1 (c) 2018 Harborside $^{\text {TM }}$ $\mathrm{s}$ a member of the American Society of Clinical Oncology (ASCO), I occasionally receive emails containing requests for survey participation. This morning, the survey requested my thoughts on how I address obesity, physical activity, and nutrition with my patients. I confess this topic was on my mind. I had recently returned from a vacation to Kauai where I saw many people on airplanes and in the airport and had been struck by the number of overweight individuals. My first thought was to wonder if people are aware of the cardiovascular and cancer risks associated with obesity. But after the ASCO survey this morning, my thoughts are of my role in tackling the very real risks of cancer associated with obesity in my patients. Am I doing enough to educate my patients, and when confronted with obesity in my patient population, am I addressing the issue realistically with them?

\section{CANCER AND OBESITY: THE FACTS}

Cancer is a major cause of death in our population. However, for adults, many of these cancers are attributable to modifiable risk factors (such as cigarette smoking, secondhand smoke, alcohol intake, consumption of red and processed meats, exposure to ultraviolet radiation, low consumption of fruits and vegetables, low dietary fiber, and six cancer-associated infections; Islami et al., 2017). When studying adults aged 30 years and older in the United States in 2014, researchers found that cigarette smoking accounted for the highest proportion of cancer cases (19\%), but that the next highest cause of cancer cases was attributed to excess body weight (7.8\%). These numbers matter.

In the study, excess body weight was associated with $4.8 \%$ of all cancers in men and $10.9 \%$ of all cancers in women (Islami et al., 2017). Obesity accounted for more than one-half of all uterine cancers and one-third of gallbladder, liver, and kidney/renal pelvis cancers in women. Obesity was also a factor in esophageal and gastric cancers, with a higher number of cases in men than in women (Islami et al., 2017). Obesity is connected to changes in metabolism and hormones, and with chronic inflammation, may be a factor in the development of cancer (Colditz \& Peterson, 2018). When obesity was combined with alcohol intake, poor diet, and 
physical inactivity, these factors accounted for the highest proportion of all cases of cancer in women and followed tobacco smoking (the highest factor) for cases in men. The most glaring fact about these statistics for me remains that these factors are modifiable. We can do something about them, and yet obesity remains a huge problem for our population.

\section{ROLE OF THE}

\section{ADVANCED PRACTITIONER}

This brings me back to my role as an advanced practitioner working with patients and their families to address health-care needs. The ASCO survey asked me to quantify how many times I assess a patient's body mass index during a visit and asked if I advise patients on their physical activity, diet, and need to increase or lose body weight. There were questions regarding referrals for weight management and visits to dieticians. Barriers to discussions of healthy weight included the lack of time to set up a referral or to do counseling, reimbursement, patient resistance, and lack of training. Although the survey was short, it did prod my thinking about my role in helping my patients achieve and maintain a healthy weight. It also made me think about my role in educating my patients and their families regarding the very real risk obesity plays in the development of cancer. I have to confess that the association between weight and cancer is not the first thing I think of during a 20-minute visit to examine a patient for appropriateness of treatment, but it should certainly be a part of the discussion, and should be of particular importance in the follow-up care after active therapy.

So why are discussions of optimal weight and risks of cancer sometimes difficult to initiate? It's clear that obesity plays a very important role in the development of cancer, and yet discussion of weight seems so personal. I've had little problem in discussing smoking cessation with patients, and yet I can remember initiating a discussion of body weight with a patient with breast cancer and having the patient smile disarmingly at me, telling me, "All the girls in my family are bigboned...that's just the way we are." I've shared a weight-loss handout to patients and found the pamphlet left in the waiting room area. Weight loss is a subject that many people don't want to talk about, or find it too personal to discuss with a health-care practitioner.

But discussion is needed. The statistics are alarming. If more than $40 \%$ of cancer cases in America can be prevented, and obesity is the second highest modifiable cause of cancer in both sexes, we need to initiate these discussions. Presently, three-fourths of Americans are considered overweight or obese. Obesity is responsible for $7.8 \%$ of cancer cases in both sexes and $6.5 \%$ of deaths. Lifestyle modifications to include weight loss can make a difference. But as a health-care provider, my responsibility is to address the need for a healthy lifestyle change with my patients by increasing my comfort level and beginning the discussion.

\section{References}

Colditz, G. A., \& Peterson, L. L. (2018). Obesity and cancer: Evidence, impact, and future directions. Clinical Chemistry, 64(1), 154-162. https://doi.org/10.1373/ clinchem.2017.277376

Islami, F., Sauer, A. G., Miller, K. D., Siegel, R. L., Fedewa, S. A., Jacobs, E. J.,...Jemal, A. (2017). Proportion and number of cancer cases and deaths attributable to potentially modifiable risk factors in the United States. $C A: A$ Cancer Journal for Clinicians, 68(1), 31-54. https://doi. org/10.3322/caac. 21440 